\title{
Photospheric Sources and Brightening of the Internetwork Chromosphere
}

Article in The Astrophysical Journal · December 2008

DOI: $10.1086 / 367833$

CITATIONS

8

4 authors, including:

\section{Ana Cadavid}

California State University, Northridge

98 PUBLICATIONS 878 CITATIONS

SEE PROFILE

\section{Alexander Ruzmaikin}

California Institute of Technology

400 PUBLICATIONS 5,079 CITATIONS

SEE PROFILE
READS

30

\section{John K. Lawrence}

California State University, Northridge

147 PUBLICATIONS 1,091 CITATIONS

SEE PROFILE 


\title{
PHOTOSPHERIC SOURCES AND BRIGHTENING OF THE INTERNETWORK CHROMOSPHERE
}

\author{
A. C. Cadavid and J. K. Lawrence \\ Department of Physics and Astronomy, California State University, Northridge, CA 91330-826; ana.cadavid@csun.edu
}

T. E. BERGER

Lockheed Martin Solar and Astrophysics Laboratory, Palo Alto, CA 94304

AND

A. RUZMAIKIN

Jet Propulsion Laboratory, California Institute of Technology, Pasadena, CA 91109

Received 2002 October 24; accepted 2002 December 7

\begin{abstract}
We analyze a unique $9 \mathrm{hr}$ sequence of near-simultaneous, high-resolution and high-cadence $G$-band and $\mathrm{K}$-line solar filtergrams, together with magnetograms of lower cadence and resolution. Our focus is on the phenomena surrounding discrete photospheric darkening "events" in internetwork $G$-band intensities. $72 \%$ of the darkenings are followed after 2 minutes by K-line brightenings. In the remaining cases, the darkenings are instead preceded by K-line brightenings 2 minutes earlier. Equivalent results are found when reference is shifted to K-line brightening events, although these two sets overlap by no more than $15 \%$. The timing and coupling of the photospheric darkenings and chromospheric brightenings appear to be regulated by a preexisting 4 minute oscillation of the solar atmosphere. Other oscillations with periods in the range $1-8$ minutes also are present, and in general the wave power is doubled at the time of an event. Our results favor an acoustic source for enhanced amplitudes of K-line intensity oscillations. The magnetic field acts as a passive tracer of horizontal photospheric flows that converge on the photospheric darkening events and then rebound.
\end{abstract}

Subject headings: Sun: chromosphere — Sun: magnetic fields — Sun: oscillations — Sun: photosphere

\section{INTRODUCTION}

The study of the heating and related dynamics of the quiet chromosphere has a long history (Rutten \& Uitenbroek 1991). The properties of oscillations present in the signals of $\mathrm{K}_{2 V}$ bright points have been extensively investigated, as well as the relation of such brightenings to the weak magnetic fields present in the internetwork regions. It has been found that observed waves have periods primarily in the range $2-5$ minutes, with the majority of cases showing a 3 minute oscillation (e.g., Orrall 1966; Liu 1974; Cram 1978). There have been different attempts to explain the $\mathrm{Ca}$ II brightenings. These include 3 minute period acoustic waves (Carlsson \& Stein 1992; Kalkofen 1996), 5 minute oscillations (Kalkofen et al. 1992), and short-period waves (Stein 1968). Others have suggested shocks (Ulmschneider 1970, 1974; Rammacher \& Ulmschneider 1992) or an extended wave pattern characterized by beats (von Uexküll \& Kneer 1995). A possible theoretical explanation was put forward by Carlsson \& Stein (1997), who simulated the generation of realistic $\mathrm{Ca}$ II $\mathrm{H}_{2 V}$ bright grains by acoustic shocks alone. Krijger et al. (2001) identified upward propagation for waves with periods $2-5$ minutes, as well as the origin of acoustic shocks in the upper photosphere. They also suggested that there is an additional contribution to the process of bright grain formation due to a low-frequency modulation of the atmosphere caused by internal gravity waves.

Most investigators have found no significant correlation between photospheric magnetic fields and the overlying bright points (Nindos \& Zirin 1998; Lites, Rutten, \& Berger 1999; Worden, Harvey, \& Shine 1999). However, Sivaraman et al. (2000) indicated a high spatial correlation between $\mathrm{Ca} \mathrm{K}_{2 V}$ bright points and magnetic elements in simultaneous K-line spectroheliograms and magnetic images. Also, McIntosh et al. (2001) have found evidence of propagating magnetoacoustic waves.

If indeed the scenario described by Carlsson \& Stein (1997) is correct, it is essential to describe the acoustic "piston" that initiates the waves in the photosphere. Hoekzema \& Rutten (1998) found that bright chromospheric pixels in $\mathrm{Ca}$ II $\mathrm{K}_{2 V}$ filtergrams occur preferentially over dark lanes in $G$-band photospheric images, with the Kline events occurring about 2.5 minutes after the $G$-band observations. Rimmele et al. (1995), Goode et al. (1998), and Strous, Goode, \& Rimmele (2000) suggested that peaks in the vertical acoustic flux could act as sources for the waves. These "acoustic events" were localized in the intergranular lanes. Goode (2002) went further and, for sites near the solar surface with weak magnetic fields present, found a connection between "seismic events" in the dark intergranular lanes and catastrophic collapses of the lanes. Chromospheric bright points seem to occur above and, a short time after, the large seismic events. In recent work Hoekzema, Rimmele, \& Rutten (2002) found that sites in the photosphere with the largest upward acoustic flux have a larger than random probability of preceding very bright chromospheric grains by an average time of 2 minutes. Skartlien, Stein, \& Nordlund (2000), using a threedimensional convection model that extends to the chromosphere, identified as the sources of the acoustic events, sites in the intergranular lanes characterized by collapsing small granules and the reversal of upflows into downflows.

There are still open questions in the understanding of the origins of the internetwork chromospheric brightenings. In particular, what are the properties of the dark intergranular lane events and their coupling to chromospheric brightenings, and what might be the role of the magnetic field in the process? In this work we set out to investigate the 
first question by studying the link between acoustic events, as indicated by intergranular lane darkenings, to chromospheric heating, as indicated by $\mathrm{Ca}$ II filtergrams. This is done by analyzing a unique data set that consists of nearly simultaneous, high-resolution, high-cadence, and longduration $G$-band and K-line observations. In addition, we attempt to address the second question with magnetogram data of lesser resolution and lower cadence.

Section 2 describes the data, while $\S 3$ presents the results of the analysis. Section 4 gives a summary of the observational results and discusses how they support or contradict different models.

\section{DATA}

The data were taken on 1998 May 30 from UT 07:50 to 17:40 with the Swedish Vacuum Solar Telescope (SVST) using $G$-band and $\mathrm{Ca}$ II $\mathrm{K}$-line interference filters and the Lockheed SOUP instrument. Details on the instrumentation and data processing can be found in Berger \& Title (2001). The data used in the present analysis consist of a subset of the original images, corresponding to a region of quiet Sun (internetwork) near the disk center. The sequence has 1541 images that include cospatial, nearly simultaneous $G$-band (4305 $\AA$, bandpass $12 \AA$ ) and Ca II K (3934 А, bandpass $3 \AA$ ) filtergrams. The cadence of the $G$-band and $\mathrm{Ca} \mathrm{K}$ observations is $\sim 21$ s. Cospatial Magnetograms Fe I (6302 $\AA$ ) are also available but with a cadence of $\sim 90$ s. The filtergram resolution is $\geq 0.2 \mathrm{Mm}$, and that of the magnetograms is $\geq 0.4 \mathrm{Mm}$. The pixel scale is $0.06 \mathrm{Mm}$. The single-pixel magnetogram sensitivity is less than $225 \mathrm{Mx} \mathrm{cm}^{-2}$ (Berger \& Lites 2002). The images were co-registered by us to 1-2 pixel accuracy.

Figures $1 a-1 c$ is intended to give a general characterization of the original data set. It shows nearly simultaneous examples of a $G$-band filtergram, a Ca II K-line filtergram, and the average of four magnetograms of the same region. The ovals show regions of $\mathrm{kG}$ magnetic elements that result in distinct high-brightness patterns in the K-line chromospheric image. In addition, $G$-band bright points are associated with the rightmost pair of regions. In contrast, the rectangle outlines one of the transitory $\mathrm{K}$-line brightenings that is not associated with any detectable magnetic flux in the magnetogram (noise level approximately $60 \mathrm{Mx} \mathrm{cm}^{-2}$ ) but is centered on the very dark intergranular region at the intersection of several granules.

Finally, a $4 \times 4$ pixel average was performed so that the new pixel scale of $0.24 \mathrm{Mm}$ matches the resolution and the per pixel magnetogram sensitivity improves to less than 60 $\mathrm{Mx} \mathrm{cm}{ }^{-2}$. The internetwork subregion used in the present analysis essentially has a field of view of $24 \times 14 \mathrm{Mm}$ on the Sun. The exact outline is given in Figures $2 a-2 c$, which include $G$-band, K-line, and magnetogram images in the larger pixel scale. This internetwork subregion was used throughout the $9 \mathrm{hr}$ data sequence with no additional masking. However, we note that the procedure given below for selecting photospheric darkening events is effectively self-masking.

\section{RESULTS}

We analyze the data set to relate changes in the photosphere, represented by $G$-band and magnetic images, to effects in the chromosphere, represented by the K-line
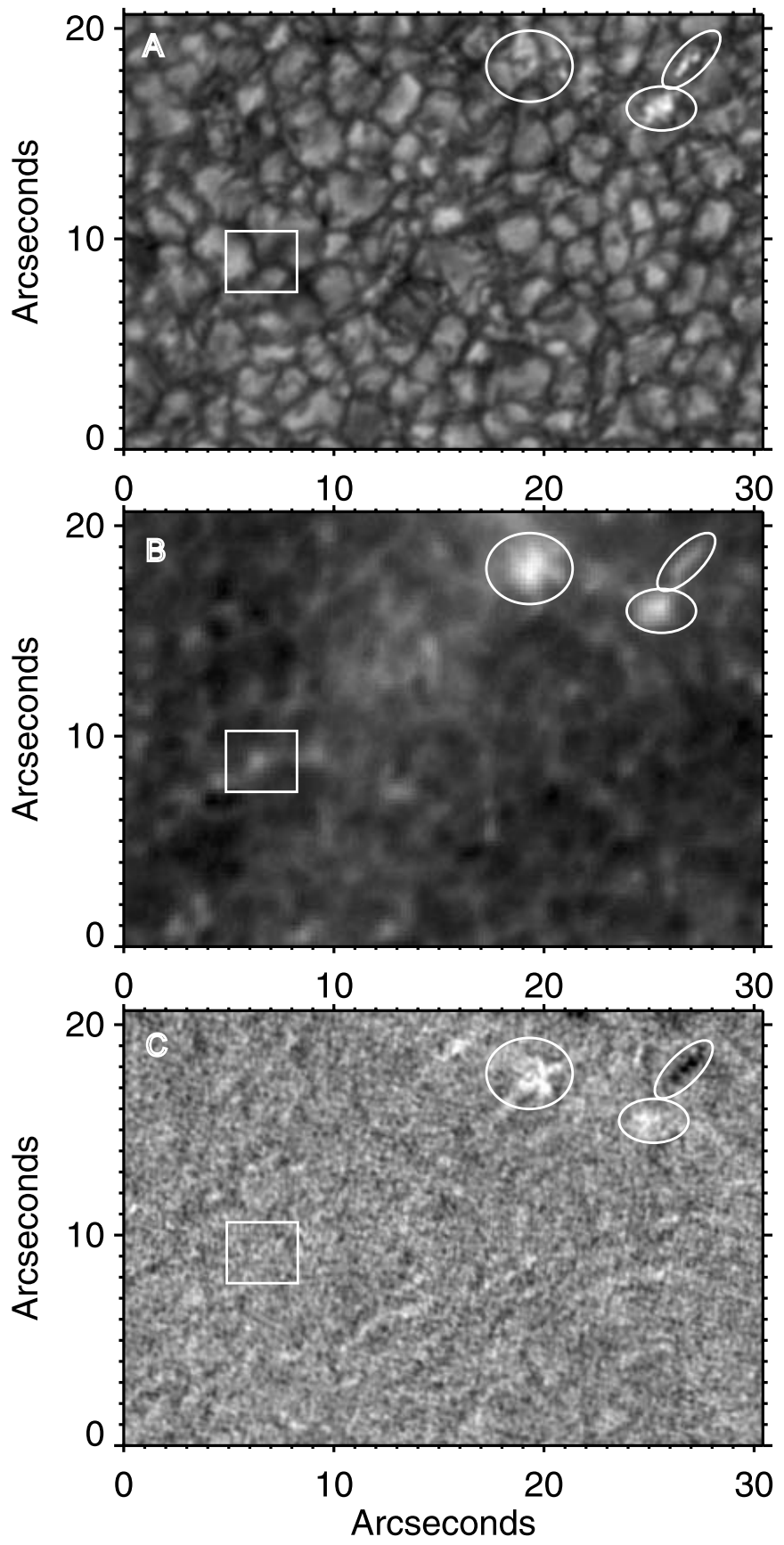

FIG. 1.-Representative images from the 1998 May 30 SVST data set. (a) $G$-band $4305 \AA$ filtergram of a relatively quiet region taken at 09:29:47 UT. (b) Ca II K-line filtergram of the same region taken at 09:29:50 UT. (c) Average of four Fe I $6302 \AA$ magnetograms of the same region, with the first image taken at 09:23 UT and the last taken at 09:29 UT. The ovals show regions of $\mathrm{kG}$ magnetic elements that result in distinct highbrightness patterns in the K-line chromospheric image. In addition, $G$-band bright points are associated with the rightmost pair or regions. In contrast, the rectangle outlines one of the transitory $\mathrm{K}$-line brightenings, which is not associated with any detectable magnetic flux in the magnetogram (noise

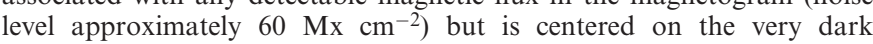
intergranular region at the intersection of several granules.

images. In particular, we investigate changes related to discrete darkening events in intergranular lanes in the quiet photosphere. These discrete darkenings, associated with downflows in the boundaries of the granulation pattern, have been previously identified as "intergranular holes" by Roudier et al. (1997). Working with the internetwork 

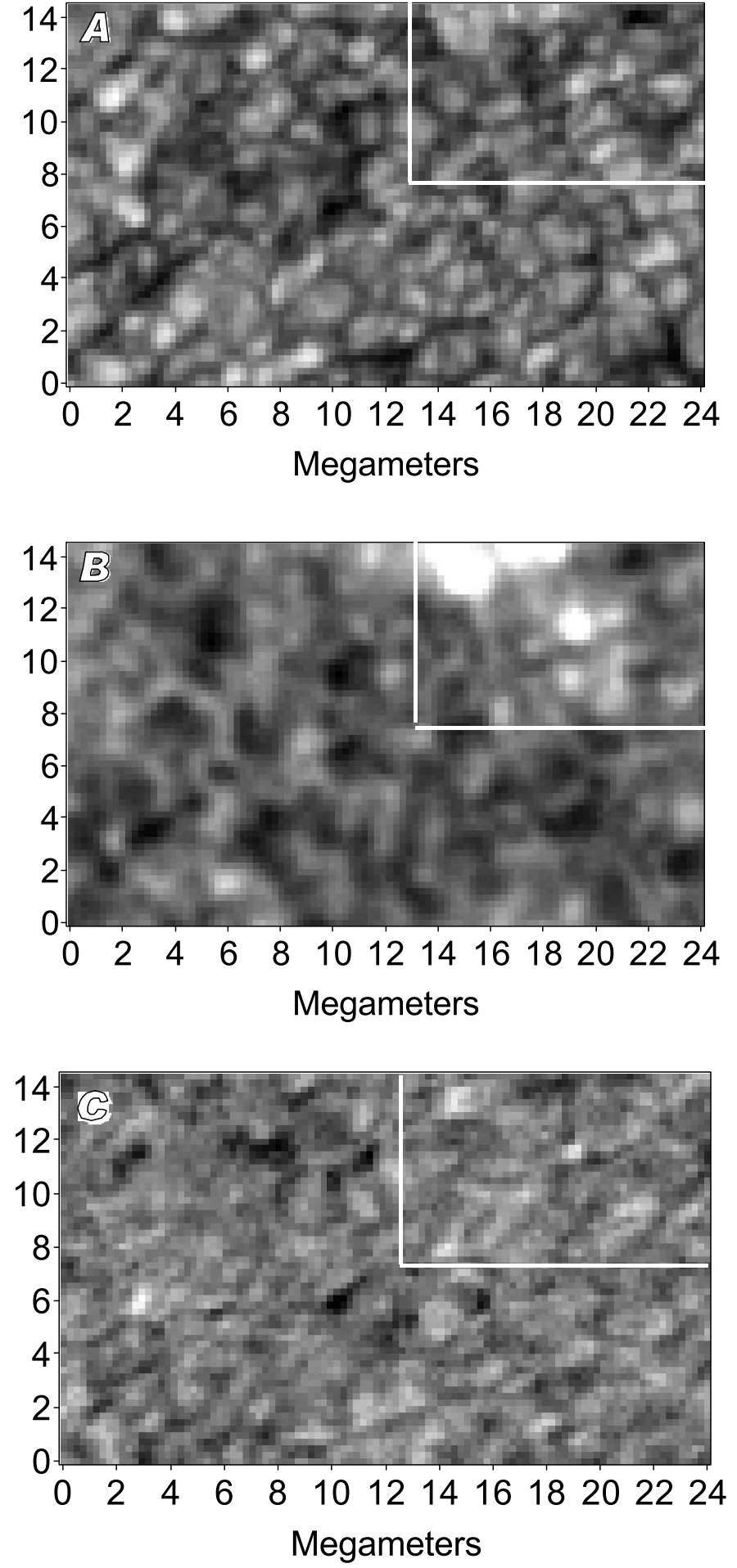

FIG. 2.-Internetwork subregions obtained by performing a $4 \times 4$ pixel average that leads to a pixel scale of $0.24 \mathrm{Mm}$. The outline describes the region used in the analysis with the top right rectangle excluded. (a) $G$-band filtergram, $(b) \mathrm{K}$-line filtergram, and $(c)$ corresponding magnetogram.

subregion described in $\S 2$, we have searched the whole sequence of $G$-band images for local minima representing the lowest intensity within a spacetime volume $1.2 \mathrm{Mm} \times$ $1.2 \mathrm{Mm} \times 10$ minutes centered on the point of interest. We will henceforth refer to these as darkening, or $G$-band, or photospheric "events." Note that these events are selected according to their spacetime isolation, not their degree of darkness. Nevertheless, we find that they do include the darkest points of the data set. We found 1527 darkening events, and at the spatial location of each, we established a relative time coordinate $t_{G}$ with $t_{G} \equiv 0$ at the time of the event. For each darkening event, we determined the $G$-band and K-line intensities and the absolute value of the line-of-sight magnetic field $\left|B_{z}\right|$ as functions of $t_{G}$ at the same spatial location. These are then averaged over all events within time bins. In order to plot the three quantities together, we standardize the units by subtracting from each bin the overall time average of the data and then dividing by the corresponding standard deviation, $I \rightarrow\left(I-\langle I\rangle_{t}\right) / \sigma_{I t}$.

Figure 3 shows the mean $G$-band intensity, mean K-line intensity, and the mean magnetic field magnitude as functions of $t_{G}$ in standardized units. The error bars are $1 \sigma$ uncertainties (standard deviations of the mean in each time bin). The mean $G$-band intensity shows a strong, sharp dip centered on the time of the $G$-band darkening event at $t_{G}=0$. The corresponding mean $\mathrm{K}$-line intensity oscillates in time. At $t_{G}=-1.7$ minutes there is a small maximum, followed by a strong minimum, that coincides with the minimum in $G$-band intensity. This is followed by a large $\mathrm{K}$-line maximum at $t_{G}=1.8$ minutes. This leads us naturally to define a K-line brightening caused by a particular photospheric darkening event to have occurred when there is an increase in $\mathrm{K}$-line intensity from $\sim 2$ minutes before the photospheric event to $\sim 2$ minutes after. The curves displayed in Figure 3 are averages over all events. To further illustrate the phenomenon, Figure 4 shows the $G$-band and $\mathrm{K}$-line light curves of one " typical" realization. Here we see the $G$-band darkening followed 2 minutes later by the maximum K-line intensity. Note that in both Figures 3 and 4 the $\mathrm{K}$ line darkens with the $G$ band, typically reaching its minimum 10-12 s later. This is not an artifact of the observations, since there is no systematic relationship between the

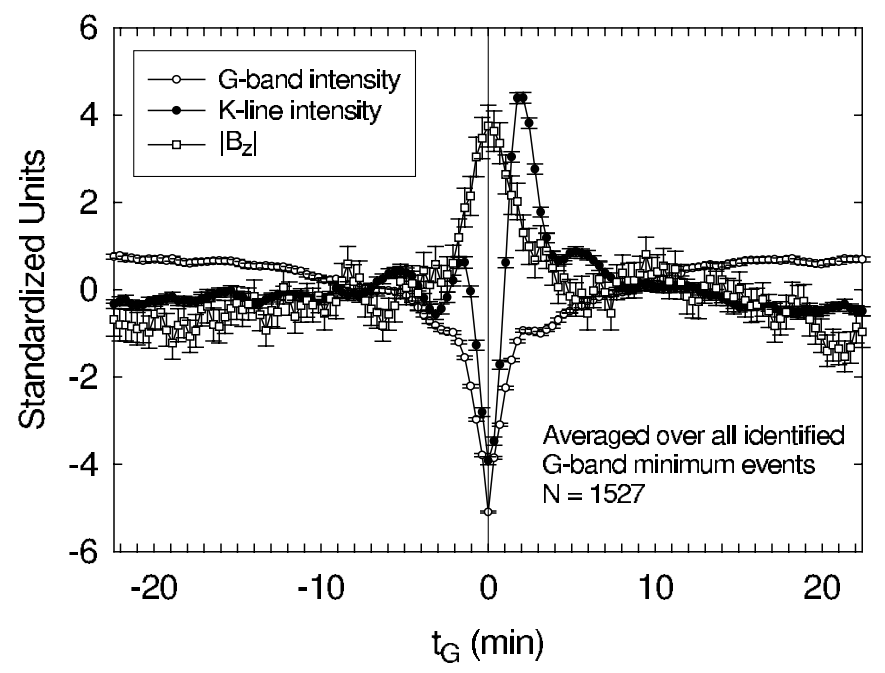

FIG. 3.-Event-averaged $G$-band intensity, K-line intensity, and magnetic field magnitude as functions of $t_{G}$, the time about a photospheric event. At about 10 minutes before the event, the $G$-band intensity starts a slow decay that steepens at $t_{G}=-2$ minutes. After reaching the minimum intensity, the process is reversed, with a fast increase up to $t_{G}=+2$ minutes and subsequent slow growth. The mean K-line intensity oscillates in time, showing a small maximum at $t_{G}=-1.7$ minutes, followed by a minimum that coincides with the minimum in $G$-band intensity, and a large maximum at $t_{G}=+1.8$ minutes. The magnetic flux accumulates at $t_{G}=0$ minutes and dissipates within $\sim 5$ minutes. 


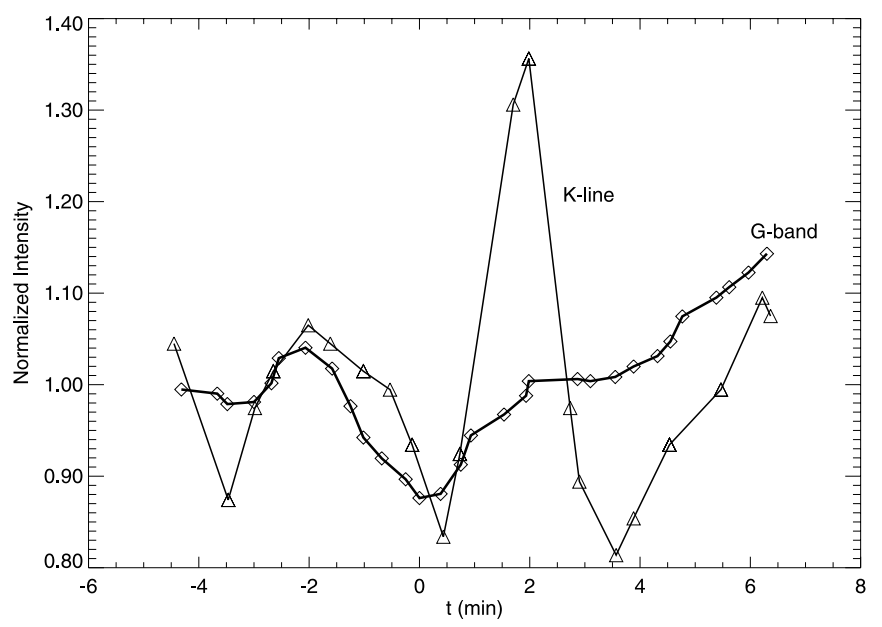

FIG. 4.- Light curve of a particular " typical" event showing the $G$-band darkening, followed 2 minutes later by the maximum K-line brightening. There is an underlying wave that modulates and synchronizes the $G$-band and $\mathrm{K}$-line fluctuations.

times at which the $\mathrm{K}$-line and $G$-band images were exposed. Note also that the mean $G$-band intensity shows weak upturns at the time of the K-line maxima near $t_{G} \sim \pm 2$ minutes. These results suggest that there is an underlying oscillation of period $\sim 4$ minutes that modulates the $G$-band and $\mathrm{K}$-line intensities. In addition, a photospheric event may stimulate an additional increase in the K-line intensity at the next maximum. Figure 3 also shows that on the average, magnetic flux accumulates at the times and places of the photospheric events and dissipates within $\sim 5$ minutes thereafter. The two maxima in the $\mathrm{K}$ line occur in the increasing and decreasing stages of the magnetic field. This suggests the presence of photospheric inflows that converge and then diverge at photospheric darkenings and transport the flux as a passive tracer.

Figure 3 shows that, on the average, $G$-band darkening events lead to K-line brightenings. But is this true in every case? The curves in Figure 3 are averages over all 1527 photospheric events. In Figure 5 we have segregated these into two groups. Those in the first group (1098, or $72 \%$ ) present an increase in K-line peak intensity after the photospheric event. Those in the second group (429, or $28 \%$ ) do not, and essentially all of these present decreases. This indicates that in almost $30 \%$ of the cases, the photospheric events are preceded, rather than followed, by strong K-line brightenings. For these cases, Figure 5 shows that the mean $G$-band intensity has a precursor local minimum at $t_{G}=-3.5$ minutes followed by the "true" minimum at $t_{G}=0$. For the first, $72 \%$ group, the magnetic field peaks $\sim 20 \mathrm{~s}$ after the photospheric event, while for the second, $28 \%$ group, the magnetic field peaks $\sim 20 \mathrm{~s}$ before. The difference in the magnitudes of the magnetic field maxima is not statistically significant. The magnetic field dissipates at a faster rate $(\sim 2$ minutes $)$ for the cases without brightenings after the photospheric event than for those with brightenings ( $\sim 5$ minutes). The magnetic influence, if any, on whether photospheric events are followed or preceded by K-line brightenings appears to be very weak.

In the next step of the analysis, we shifted the focus to the chromosphere and isolated chromospheric brightening events or brightenings by searching the whole sequence

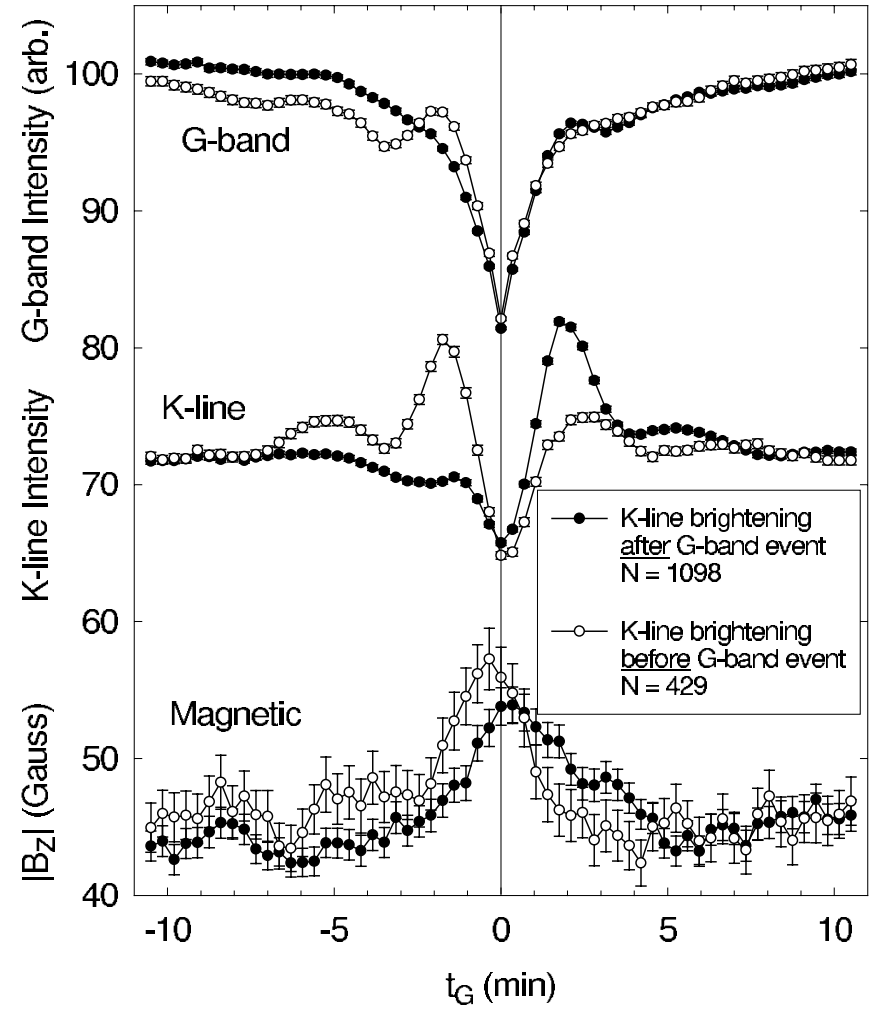

FIG. 5.-Event-averaged $G$-band intensity, K-line intensity, and magnetic field magnitude as functions of $t_{G}$. Closed circles: Events with K-line brightening after $G$-band minimum. The magnetic field peaks $\sim 20$ s after the photospheric event. Open circles: Events without K-line brightening after $G$-band minimum. The maximum in K-line intensity occurs before the photospheric event and after a local precursor minimum in $G$-band intensity at $t_{G}=-3.5$ minutes. The magnetic field peaks $\sim 20 \mathrm{~s}$ before the photospheric event.

of $\mathrm{K}$-line images for local maxima corresponding to the highest intensity within a spacetime volume $1.2 \mathrm{Mm} \times$ $1.2 \mathrm{Mm} \times 10$ minutes centered on the point of interest. We found 1465 such brightening events and, as before, at the spatial location of each, we establish a relative time coordinate $t_{\mathrm{K}}$ with $t_{\mathrm{K}} \equiv 0$ at the time of the event. For each event, we determine the K-line and $G$-band intensities and the magnetic field $\left|B_{z}\right|$ at the same spatial location as functions of $t_{\mathrm{K}}$ (Fig. 6). Once again, the event-averaged $\mathrm{K}$-line and $G$ band curves present oscillatory modulation. The K-line maximum is flanked by local minima at $t_{\mathrm{K}}= \pm 2$ minutes. These minima correspond to darkenings in the $G$ band. The magnetic field presents a lesser maximum at $t_{\mathrm{K}}=-2$ minutes, corresponding to the first, deepest $G$-band minimum. The greater maximum at $t_{\mathrm{K}}=1$ minute comes while both the mean $G$-band and $\mathrm{K}$-line intensities are dropping toward the second minimum. The magnetic pulse dissipates in $\sim 3$ minutes. The $\mathrm{K}$-line maximum occurs as the magnetic field is growing toward its second, greater maximum.

We next proceeded to separate the events according to whether the K-line brightening event was preceded by a deep $G$-band darkening (1063 cases or $73 \%$ ) or not (402 or $27 \%$ ). As shown in Figure 7, the magnetic field maximum before the event, at $t_{\mathrm{K}}=-2$ minutes, corresponds to those points that have a deep darkening also preceding the chromospheric brightening, while the magnetic field maximum at $t_{\mathrm{K}}=1$ minute corresponds to the points with a deep darkening afterward. This indicates that the degree of 


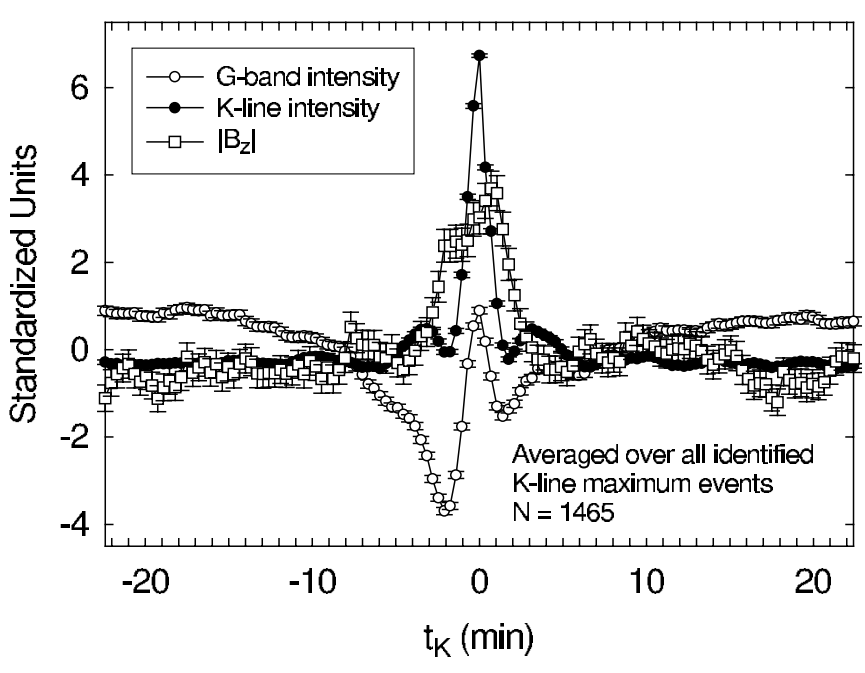

FIG. 6.-Event-averaged $G$-band intensity, K-line intensity, and magnetic field magnitude as functions of $t_{\mathrm{K}}$, the time about a chromospheric event. The peak in the $\mathrm{K}$ line is flanked by minima at $t_{\mathrm{K}} \pm 2$ minutes, which coincides with darkenings in the $G$ band. The magnetic field presents a lesser maximum at $t_{\mathrm{K}}=-2$ minutes and a greater maximum at $t_{\mathrm{K}}=1$ minute. The magnetic pulse dissipates in $\sim 3$ minutes.

accumulation of magnetic field is determined by the strength of the darkening, which is probably related to the strength of the flow converging to the intergranular site. This result further confirms the notion that the magnetic field is acting as a passive flow tracer.

The two types of events described above result from different definitions. In one case, the photospheric events describe 1527 local $G$-band minima and correspond to deep darkenings in the photosphere that, to some extent, act as triggers for a chromospheric brightening. In the second case, the 1465 chromospheric brightening events are defined as K-line local maxima that correspond to internetwork chromospheric brightenings. Comparison of the two sets of events indicates that only $10 \%-15 \%$ of the events are

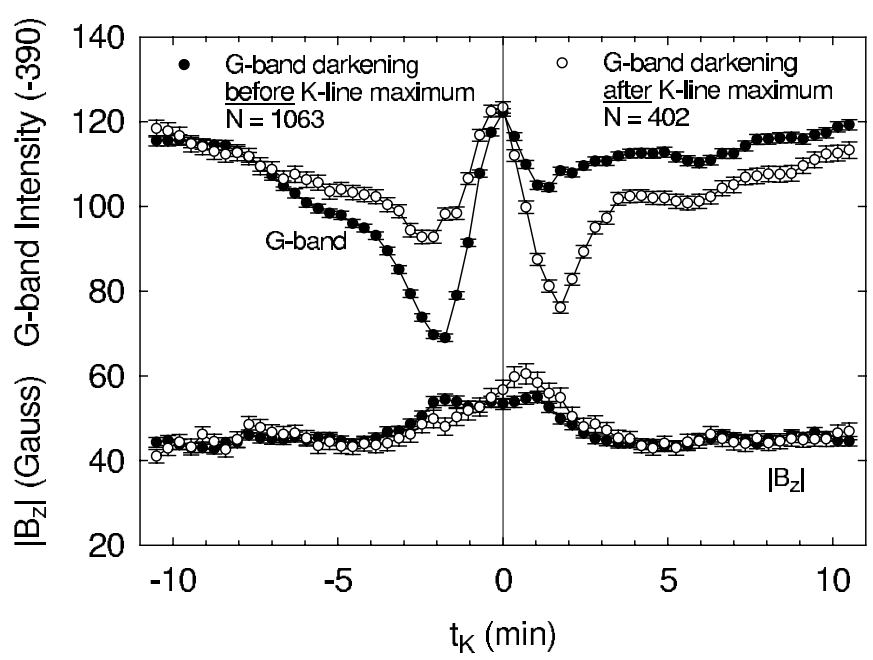

FIG. 7.-Event-averaged K-line intensity and magnetic field magnitude as functions of $t_{\mathrm{K}}$. Closed circles: Events with $G$-band darkening before $\mathrm{K}$ line maximum. The magnetic field has a maximum before the event at $t_{\mathrm{K}}=-2$ minutes. Open circles: Events without $G$-band darkening before $\mathrm{K}$-line maximum. The magnetic field has a maximum after the event at $t_{\mathrm{K}}=1$ minute. common to both populations (depending on the tolerance in defining commonality). If the events were randomly distributed in space and time, we would expect less than $0.5 \%$ overlap. This indicates that although the two sets are related, they are far from coinciding. This is in agreement with the finding of Hoekzema et al. (2002).

The data averaging process leading to Figures 3, 5, 6, and 7 can result in cancellations in signed $G$-band and K-line fluctuations as one moves away in time from the events. If there are long periods of coherent oscillations (von Uexküll \& Kneer 1995) with slightly different periods or phases from one realization to the next, this information will be lost. In order to avoid this problem and to further investigate the properties of the waves in the chromosphere, we have calculated the wavelet power in the K-line signal for periods of $1-$ 8 minutes (the Nyquist limit is 0.7 minutes). A continuous transform with complex Morlet basis function (Torrence \& Compo 1998) ${ }^{1}$ was used. The average power at the photospheric darkening events is shown as a function of $t_{G}$ in Figure 8 . Because the wavelet power is positive, no cancellation can occur during averaging. Figure 8 shows that, on average, there is indeed power at these periods at all times before and after the events. The 1 minute power is an order of magnitude lower than that at longer periods; the power rises quickly longward of 1.8 minutes. The wavelet power at all of these periods is doubled near the photospheric events. The maximum enhanced power occurs for period $\sim 4$ minutes. For all periods, the peak appears $\sim 1$ minute after

${ }^{1}$ Additional information can be found at http://paos.colorado.edu/ research/wavelets.

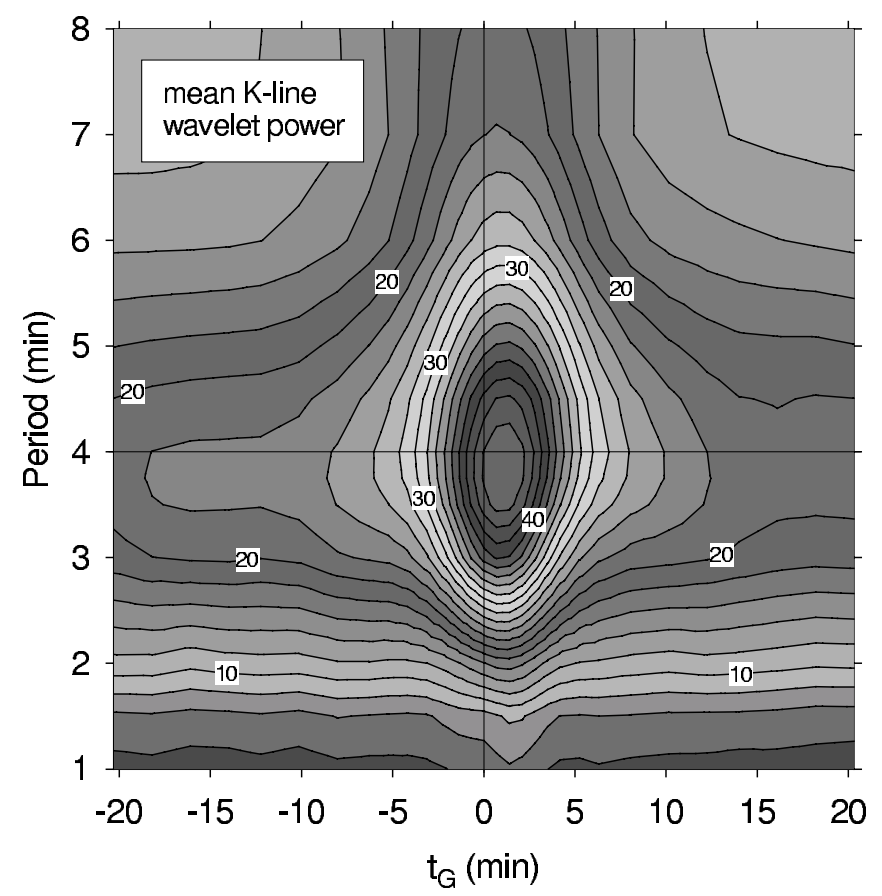

FIG. 8.-Contour plot of the event-averaged K-line wavelet power, for periods in the range 1-8 minutes, as a function of $t_{G}$, the time about a photospheric event. The 1 minute power is an order of magnitude lower than that at longer periods. The maximum enhanced power occurs for a period of $\sim 4$ minutes. The widths of the enhancement peaks for long periods depend on the period. 


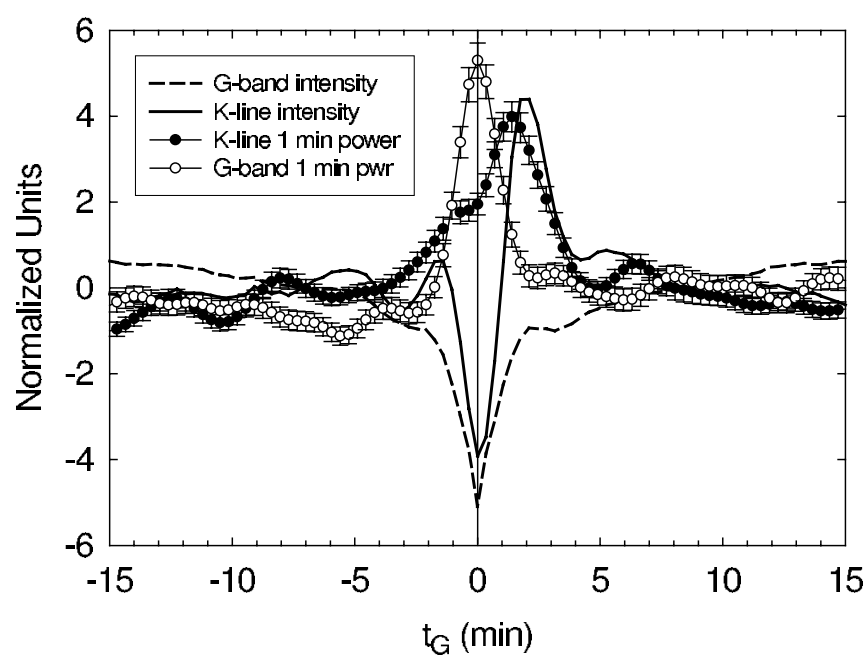

FIG. 9.-Event-averaged $G$-band intensity, K-line intensity, $G$-band 1 minute power, and K-line 1 minute power as functions of $t_{G}$, the time about a photospheric event. The K-line power presents a shoulder at $t_{G}=-1$ minute and peak value at $t_{G}=1.5$ minutes. The $G$-band 1 minute power peaks at $t_{G}=0$ minutes and is contained between $t_{G}= \pm 2$ minutes.

the photospheric event, i.e., between the event and the subsequent $\mathrm{K}$-line maximum. The widths of the enhancement peaks for long periods are proportional to their periods, which indicates that the peaks are not resolved. However, structure within the peaks can be resolved for the 1 and 2 minute pulses. In Figure 9 we show the $G$-band and K-line 1 minute wavelet power, together with the corresponding intensity curves. The $\mathrm{K}$-line power begins to increase at $t_{G}=-4$ minutes, presents a shoulder at $t_{G}=-1$ minute, and reaches its peak value at $t_{G}=1.5$ minutes. This occurs before the K-line intensity peak at $t_{G} \sim 2$ minutes. The power then drops at a faster rate, returning to its level before the event at $t_{G}=4$ minutes. The $G$-band 1 minute power peaks at $t_{G}=0$ minutes and is contained between $t_{G}= \pm 2$ minutes.

When the data were once again segregated according to whether a K-line brightening followed or preceded the photospheric darkening events, we found that the shoulder in the mean $\mathrm{K}$-line 1 minute wavelet power at $t_{G}=-1$ minute is in fact the dominant peak for the smaller subset of events in which the K-line maximum precedes the photospheric event. This wavelet peak follows the weak precursor $G$-band minimum at $t_{G}=-3.5$ minutes and also follows the K-line maximum at $t_{G}=-2$ minutes, which were shown in Figure 5 . For both classes of points the maximum of 1 minute $G$-band power occurs as the $G$-band intensity reaches its minimum at $t_{G}=0$.

A study of phase lags between the K-line and $G$-band wavelets indicated essentially no propagation (lag $12 \mathrm{~s}$ ) at periods 3-5 minutes, suggesting evanescent waves (Fleck \& Schmitz 1991; Kalkofen 1991). On the average, there was upward propagation for period $\sim 1$ minute (frequency $\sim 17$ $\mathrm{mHz}$ ) waves, but statistics were insufficient to determine a flux.

\section{SUMMARY AND DISCUSSION}

Here we summarize the results we have found and compare them with some suggested mechanisms for K-line brightenings.
Some of our results are reinforced and complemented by recent work of Hoekzema et al. (2002). Unlike ours, their data permit the simultaneous determination of Doppler shifts at multiple heights in the solar atmosphere and hence the calculation of an acoustic flux. However, their image cadence of $47 \mathrm{~s}$ versus our $21 \mathrm{~s}$ limits temporal resolution. These workers compare spatiotemporally localized enhancements of acoustic wave flux with the occurrence of localized $\mathrm{K}_{2 \mathrm{~V}}$ emission in the chromosphere. They find that the instances of largest upward flux have a statistically significant (but $<1$ ) probability to be "accompanied or followed by excessive $\mathrm{Ca}$ II $\mathrm{K}_{2 V}$ oscillation amplitude in the internetwork chromosphere." Interestingly, they also find a lesser but significant amount of downward-propagating wave flux in connection with such $\mathrm{K}_{2 \mathrm{~V}}$ oscillations.

The $1527 G$-band darkening events we identify (Figs. 3 and 5) are, on the average, accompanied by transient enhancements of $\left|B_{z}\right|$. This occurs whether the darkening events are followed by K-line brightenings or are instead preceded by them. This supports the identification of the darkening events with collapsing intergranular lanes; the magnetic field acts as a passive tracer implying a photospheric inflow leading up to the $G$-band darkening event, followed by an outflow. Although it can correctly be stated that there is a strong statistical tendency for enhanced magnetic fields to be found in connection with internetwork Kline brightenings (Sivaraman et al. 2000), the dynamics of the process reveal that the magnetic fields are mere passive indicators of related photospheric flows and do not influence phenomena higher in the solar atmosphere.

The mean K-line intensity dips to a minimum at $t_{G}=0$ along with the mean $G$-band intensity (after a $12 \mathrm{~s}$ lag). The two intensities, but more strongly the $\mathrm{K}$ line, also show an in-phase 4 minute oscillation. In $72 \%$ of the $G$-band darkening events, the main $G$-band $/ \mathrm{K}$-line minima at $t_{G}=0$ are followed at $t_{G}=2$ minutes by an enhanced $\mathrm{K}$-line maximum. This is consistent with a photospheric event triggering the chromospheric brightening. However, things are not so straightforward; in the remaining $28 \%$ of the case, the $G$ band darkenings are preceded by an enhanced K-line maximum at $t_{G}=-2$ minutes. Figure 5 shows that in these cases the mean $G$-band intensity has a local precursor minimum at $t_{G}=-3.5$ minutes. Thus, there appears to be a two-way interaction between the photospheric events and a preexisting 4 minutes atmospheric oscillation. Note the downward-propagating wave flux of Hoekzema et al. (2002), mentioned above. Possibly the oscillation influences the timing of photospheric collapses. The relative timing of the photospheric darkenings and the chromospheric brightenings appears to be determined by the preexisting oscillation, at least in the cases we have identified.

Similar conclusions are reached by identifying the 1465 discrete internetwork K-line maxima and looking at the associated dynamics of the mean $G$-band and K-line intensities and magnetic field (Figs. 6 and 7). Note that no more than $15 \%$ of these internetwork $\mathrm{K}$-line maxima are associated with the photospheric darkening events meeting our selection criteria. The mean $\mathrm{K}$-line maximum at $t_{\mathrm{K}}=0$ is accompanied by a simultaneous mean $G$-band maximum. In $73 \%$ of the cases, the $\mathrm{K}$-line/ $G$-band maxima are preceded by a strong $G$-band minimum at $t_{\mathrm{K}}=-2$ minutes and followed by a weaker one at $t_{\mathrm{K}}=2$ minutes. However, in the remaining $27 \%$ of cases, on average the K-line/ $G$-band maxima are followed at $t_{\mathrm{K}}=2$ minutes by an enhanced 
$G$-band minimum and preceded by a weaker one. In both cases the preceding and following $G$-band minima are each associated with transient magnetic enhancements and thus, presumably, photospheric inflows followed by outflows. Here as before, the magnetic field appears to have no role in coupling the photospheric phenomena to the chromosphere.

Wavelet spectral analysis of K-line intensity (Figs. 8 and 9) shows the presence of significant oscillatory power at all periods of 1-8 minutes not only near identified events but at all times, although not at all places at all times. The 1 minute power is weakest; the power grows quickly for periods longward of 1.8 minutes to a maximum at period 4 minutes and then declines. Near the identified photospheric events, the mean wavelet power at all periods is roughly doubled. The maximum of the enhanced power also comes at period 4 minutes. At short periods, temporal structure is resolved (Fig. 9). The K-line power has a shoulder at $t_{G}=-1$ minute and a higher peak at $t_{G}=1.5$ minutes. The higher peak is associated with the subset of events in which the K-line brightening follows the photospheric darkening event. The shoulder becomes the dominant peak for the subset of events in which the $\mathrm{K}$-line maximum precedes the photospheric darkening event. For the latter case, the wavelet peak follows the weak precursor $G$-band minimum and the $\mathrm{K}$-line intensity maximum. In contrast, the 1 minute $G$-band power enhancement is centered at $t_{G}=0$, regardless of whether the K-line maximum precedes or follows the photospheric event. These results suggest that high-frequency $(>8$ $\mathrm{mHz}$ ) acoustic waves are associated with the events. Their exact physical interpretation is complicated, however, since they appear to propagate upward in the majority of cases but may propagate downward in others (see Hoekzema et al. 2002).

The widespread presence of wavelet power with a period near 4 minutes is consistent with the picture presented by von Uexküll \& Kneer (1995: see also Rutten \& Uitenbroek 1991; Rutten 1994, 1995) of a spacetime field of evanescent waves interacting to produce strings of chromospheric brightenings. However, in this work we have identified a set of strong, discrete photospheric darkening events that can be connected to strong enhancements of the chromospheric ( $\mathrm{K}$ line) emission. This is made clear in Figure 8 by the highly localized doublings of K-line wavelet power in association with these photospheric darkening events. On the other hand, Hoekzema \& Rutten (1998) found that bright chromospheric pixels occur primarily over dark lanes in $G$ band photospheric images with lags of about 2.5 minutes. These authors suggested a signal that propagates from the photosphere to the chromosphere in this time. Here we find that the high-frequency $(17 \mathrm{mHz}=$ period 1 minute $)$ waves peak in the $\mathrm{K}$ line about 0.5 minutes before the $\mathrm{K}$-line intensity maximum (see Fig. 9). It is thus apparent to us that the time difference between the identified photospheric darkening events and the subsequent K-line brightenings is determined by a preexisting, evanescent wave pattern with added energy becoming manifest at a time determined by its period. In effect, the amplitude of the nonpropagating 4 minute waves is increased, but its phase is not changed.
The photospheric darkenings, which act as triggering events, are compatible with the mechanism suggested by Goode (2002), in which a collapse in the intergranular lanes produces upward-propagating waves that can lead to a subsequent chromospheric brightening. To reinforce the first part of this picture, we are able to interpret transient increases of magnetic flux as representing photospheric inflow/outflow motions at these collapses. If our selected $G$-band darkenings are associated with these collapses, then we find that $72 \%$ are followed by K-line brightenings but that $28 \%$ are preceded by such brightenings. Goode noted the necessity of separating waves propagating from the events to the chromosphere from the $p$-mode field in the same region of the $k-\omega$ diagram. We are led to an alternative to the second part of this picture: that the $p$-mode field may be a physically integral part of the phenomenon.

Carlsson \& Stein (1997) suggested that acoustic shocks interacting with preexisting evanescent waves could be responsible for the production of chromospheric bright grains. They described one process in which a "smallamplitude, high-frequency wave propagates forward on the larger amplitude, evanescent, low-frequency wave and produces a shock when it catches up to the crest." Their simulations showed more generally that the "bright grains are produced primarily by waves near and slightly above the acoustic cutoff frequency. The precise time and strength of a grain depend upon the interference between these waves at the acoustic cutoff frequency and higher frequency waves. When waves near the acoustic cutoff frequency are weak, then higher frequency waves produce grains. The ' 5 minute' trapped $p$-mode oscillations are not the source of the grains, although they can modify the behavior of higher frequency waves." Various aspects of this picture fit with our findings. One is the presence of the $\sim 4$ minute underlying wave that carries the maximum power and that modulates the K-line brightenings. Another is the presence of high-frequency waves $(17 \mathrm{mHz})$ of small amplitude in the photosphere at all times, but enhanced in association with photospheric darkenings and subsequent K-line brightenings. Carlsson \& Stein (1997) note that the direct production of grains by high-frequency waves without strong waves near the acoustic cutoff is "often absent in the observations," and we do not see them here. Finally, the acoustic picture is further supported by the observation that the magnetic fields act as passive tracers of the photospheric flows that concentrate them in the intergranular lanes and that they do not seem to play a dynamic role in heating the internetwork chromosphere.

This work was supported by grants NSF-ATM 9987305 and NASA-NAG5-10880. The SVST is operated by the Swedish Royal Academy of Sciences at the Spanish Observatorio del Roque de los Muchachos of the Instituto de Astrofisica de Canarias. We are grateful to the referee, R. Rutten, for very useful comments that led to improvements of the paper.
Berger, T. E., \& Lites, B. W. 2002, Sol. Phys., 208, 181

Berger, T. E., \& Title, A. M. 2001, ApJ, 553, 449

Carlsson, M., \& Stein, R. F. 1992, ApJ, 397, L59

. 1997, ApJ, 481, 500
REFERENCES

Cram, L. E. 1978, A\&A, 70, 345

Fleck, B., \& Schmitz, F. 1991, in Proc. Int. Conf., Mechanisms of Chromospheric and Coronal Heating, ed. P. Ulmschneider, E. R. Priest, \& R. Rosner (Berlin: Springer), 22 
Goode, P. R. 2002, AAS Meeting, 200, 53.05

Goode, P. R., Strous, L. H., Rimmele, T. R., \& Stebbins, R. T. 1998, ApJ, 495, L27

Hoekzema, N. M., Rimmele, T. R., \& Rutten, R. J. 2002, A\&A, 390, 681

Hoekzema, N. M., \& Rutten, R. 1998, A\&A, 329, 725

Kalkofen, W. 1991, in Proc. Int. Conf., Mechanisms of Chromospheric and Coronal Heating, ed. P. Ulmschneider, E. R. Priest, \& R. Rosner (Berlin: Springer), 54

Kalkofen, W. 1996, ApJ, 468, L69

Kalkofen, W., Rossi, P., Bodo, G., \& Massaglia, M. 1992, in ASP Conf.

Ser. 26, Cool Stars, Stellar Systems and the Sun, ed. M. S. Giampapa \&

J. A. Bookbinder (San Francisco: ASP), 543

Krijger, J. M., Rutten, R. J., Lites, B. W., Straus, Th., Shine, R. A., \& Tarbell, T. D. 2001, A\&A, 379, 1052

Lites, B. W., Rutten, R. J., \& Berger, T. E. 1999, ApJ, 517, 1013

Liu, S.-Y. 1974, ApJ, 189, 359

McIntosh, S. W., et al. 2001, ApJ, 548, L237

Nindos, A., \& Zirin, H. 1998, Sol. Phys., 179, 253

Orrall, F. Q. 1966, ApJ, 143, 917

Rammacher, W., \& Ulmschneider, P. 1992, A\&A, 253, 586
Rimmele, T. R., Goode, P. R., Harold, E., \& Stebbins, R. T. 1995, ApJ, 444, L119

Roudier, Th., Malherbe, J. M., November, L., Vigenau, J., Coupinot, G., Lafon, M., \& Muller, R. 1997, A\&A, 320, 605

Rutten, R. 1994, in Proc. Mini Workshop, Chromospheric Dynamics, ed. M. Carlsson (Oslo: Inst. Theor. \& Astron.), 25

1995 , in Proc 4th $\mathrm{SOHO}$ Workshop, Helioseismology, ed. J. T.

Hoekzema, V. Domingo, B. Fleck, \& B. Battrick (ESA SP-376; Noordwijk: ESTEC), 151

Rutten, R., \& Uitenbroek, H. 1991, Sol. Phys., 134, 15

Sivaraman, K. R., Gupta, S. S., Livingston, W. C., Damé, L., Kalkofen, W., Keller, C. U., Smartt, R., \& Hasan, S. S. 2000, A\&A, 363, 279

Skartlien, R., Stein, R. F., \& Nordlund, A. 2000, ApJ, 541, 468

Stein, R. F. 1968, ApJ, 154, 297

Strous, L. H., Goode, P. R., \& Rimmele, T. R. 2000, ApJ, 535, 1000

Torrence, C., \& Compo, G. P. 1998, Bull. Am. Meteor. Soc., 79, 61

Ulmschneider, P. 1970, Sol. Phys., 12, 403 1974, Sol. Phys., 39, 327

von Uexküll, M., \& Kneer, F. 1995, A\&A, 294, 252

Worden, J., Harvey, J., \& Shine, R. 1999, ApJ, 523, 450 